Bereswill, Mechthild; Zühlke, Johanna

\title{
"Faktor Frau kommt meilenweit danach". Eine qualitative Exploration zum Verhältnis von Geschlecht und Behinderung
}

Budde, Jürgen [Hrsg.]; Offen, Susanne [Hrsg.]; Tervooren, Anja [Hrsg.]: Das Geschlecht der Inklusion. Opladen ; Berlin ; Toronto : Verlag Barbara Budrich 2016, S. 137-152. - (Jahrbuch Frauen- und Geschlechterforschung in der Erziehungswissenschaft; 12)

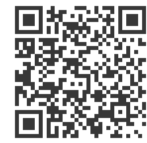

\section{Quellenangabe/ Reference:}

Bereswill, Mechthild; Zühlke, Johanna: "Faktor Frau kommt meilenweit danach". Eine qualitative Exploration zum Verhältnis von Geschlecht und Behinderung - In: Budde, Jürgen [Hrsg.]; Offen, Susanne [Hrsg.]; Tervooren, Anja [Hrsg.]: Das Geschlecht der Inklusion. Opladen ; Berlin ; Toronto : Verlag Barbara Budrich 2016, S. 137-152 - URN: urn:nbn:de:0111-pedocs-157567 - DOI: 10.25656/01:15756

https://nbn-resolving.org/urn:nbn:de:0111-pedocs-157567 https://doi.org/10.25656/01:15756

in Kooperation mit / in cooperation with:

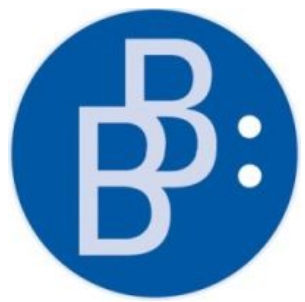

https://www.budrich.de

\section{Nutzungsbedingungen}

Gewährt wird ein nicht exklusives, nicht übertragbares, persönliches und beschränktes Recht auf Nutzung dieses Dokuments. Dieses Dokument ist ausschließlich für den persönlichen, nicht-kommerziellen Gebrauch bestimmt. Die Nutzung stellt keine Übertragung des Eigentumsrechts an diesem Dokument dar und gilt vorbehaltlich der folgenden Einschränkungen: Auf sämtlichen Kopien dieses Dokuments müssen alle Urheberrechtshinweise und sonstigen Hinweise auf gesetzlichen Schutz beibehalten werden. Sie dürfen dieses Dokument nicht in irgendeiner Weise abändern, noch dürfen Sie dieses Dokument für öffentliche oder kommerzielle Zwecke vervielfältigen, öffentlich ausstellen, aufführen, vertreiben oder anderweitig nutzen.

Mit der Verwendung dieses Dokuments erkennen Sie die Nutzungsbedingungen an.

\section{Terms of use}

We grant a non-exclusive, non-transferable, individual and limited right to using this document.

This document is solely intended for your personal, non-commercial use. Use of this document does not include any transfer of property rights and it is conditional to the following limitations: All of the copies of this documents must retain all copyright information and other information regarding legal protection. You are not allowed to alter this document in any way, to copy it for public or commercial purposes, to exhibit the document in public, to perform, distribute or otherwise use the document in public.

By using this particular document, you accept the above-stated conditions of use.

\section{Kontakt / Contact:}

\section{peDOCS}

DIPF | Leibniz-Institut für Bildungsforschung und Bildungsinformation Informationszentrum (IZ) Bildung

E-Mail: pedocs@dipf.de

Internet: www.pedocs.de

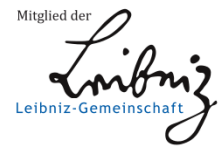


Das Geschlecht der Inklusion 
Jahrbuch

Frauen- und Geschlechterforschung

in der Erziehungswissenschaft

Redaktion

Jürgen Budde

Vera Moser

Barbara Rendtorff

Christine Thon

Katharina Walgenbach

Beirat

Birgit Althans

Sabine Andresen

Eva Breitenbach

Rita Casale

Bettina Dausien

Isabell Diehm

Hannelore Faulstich-Wieland

Edgar Forster

Edith Glaser

Carola Iller

Marita Kampshoff

Margret Kraul

Andrea Liesner

Susanne Maurer

Astrid Messerschmidt

Inga Pinhard

Annedore Prengel

Folge 12/2016 
Jürgen Budde

Susanne Offen

Anja Tervooren (Hrsg.)

\section{Das Geschlecht der Inklusion}

Verlag Barbara Budrich

Opladen • Berlin • Toronto 2016 
Bibliografische Information der Deutschen Nationalbibliothek

Die Deutsche Nationalbibliothek verzeichnet diese Publikation in der Deutschen

Nationalbibliografie; detaillierte bibliografische Daten sind im Internet über

http://dnb.d-nb.de abrufbar.

Gedruckt auf säurefreiem und alterungsbeständigem Papier.

Alle Rechte vorbehalten.

C 2016 Verlag Barbara Budrich, Opladen, Berlin \& Toronto

www.budrich-verlag.de

ISBN (Paperback) 978-3-8474-0794-2

eISBN (eBook) 978-3-8474-0922-9

Das Werk einschließlich aller seiner Teile ist urheberrechtlich geschützt. Jede Verwertung außerhalb der engen Grenzen des Urheberrechtsgesetzes ist ohne Zustimmung des Verlages unzulässig und strafbar. Das gilt insbesondere für Vervielfältigungen, Übersetzungen, Mikroverfilmungen und die Einspeicherung und Verarbeitung in elektronischen Systemen.

Typographisches Lektorat: Ulrike Weingärtner, Gründau

Umschlaggestaltung: disegno visuelle kommunikation, Wuppertal - www.disenjo.de Druck: paper \& tinta, Warschau

Printed in Europe 


\section{Inhaltsverzeichnis}

Jürgen Budde, Susanne Offen und Anja Tervooren

Das Geschlecht der Inklusion - eine Einleitung........................... 7

\section{Teil I: Essays}

Carla Di Georgio

Mothers' and Fathers' disparate experiences in Francophone Schools: The connection between language, social capital and power in accessing 'inclusive' spaces for their children .......... 15

David Mitchell, Sharon Snyder und Linda Ware

Curricular Cripistemologies: The Crip/Queer Art of Failure ... 37

Bettina Kleiner, Torben Rieckmann und André Zimpel

Diskurstheoretische Perspektiven auf Behinderung, Geschlecht und Sexualität als mögliche Grundlage der Debatte über Inklusion. Ein Versuch ............................................................. 55

Ulrike Schildmann

Von der (reflexiven) Koedukation zur (reflexiven) Inklusion ein Hürdenlauf der besonderen Art.......................................... 75 


\section{Teil II: Thementeil}

Jürgen Budde und Nina Blasse

Vergeschlechtlichungen von Care im inklusiven Unterricht.... 99

Heike Raab

Re/Visionen - Inklusion, Behinderung und Geschlecht

Mechthild Bereswill und Johanna Zühlke

„Faktor Frau kommt meilenweit danach“. Eine qualitative

Exploration zum Verhältnis von Geschlecht und Behinderung

\section{Teil III: Offener Teil}

Margarete Menz und Christine Thon

Familie und Beruf - oder? Hegemoniale Diskurse,

(un)zureichende Alternativen und die Suche nach dem ,guten

Leben"

Autor*innen 


\title{
„Faktor Frau kommt meilenweit danach“. Eine qualitative Exploration zum Verhältnis von Geschlecht und Behinderung
}

\author{
Mechthild Bereswill und Johanna Zühlke
}

\section{Einleitung}

Im Titel dieses Beitrags wird eine Sequenz aus einem Interview zitiert, das im Rahmen eines Begleitforschungsprojekts erhoben wurde (Bereswill, Pax \& Zühlke 2013: 140). Untersucht wurde ein Mentoring-Programm für Studentinnen mit Behinderung, an dem die zitierte Studentin als Mentee teilgenommen hat. Sie spricht darüber, welches Gewicht Geschlecht aus ihrer Sicht im Verhältnis zu Behinderung für die Biographien von Frauen, genauer für Akademikerinnen hat. Das Frauenförderungsprojekt des Bonner HildegardisVereins, das von 2008 bis 2013 mit Mitteln der Contergan-Stiftung gefördert ${ }^{1}$ und für drei Gruppen je ein Jahr lang durchgeführt wurde, basierte auf einem Tandem-Konzept (eine Mentee und eine Mentorin oder ein Mentor ${ }^{2}$ ), kombiniert mit Arbeitsphasen in der gesamten Gruppe. Die Beziehungsgestaltung und die Diskurse in den Gruppenprozessen standen dabei explizit wie implizit in enger Verbindung mit grundlegenden Fragen und auch konkreten Erfahrungen im Zusammenhang von Gleichstellung und Inklusion. Die meisten Teilnehmer*innen waren beruflich oder politisch in einschlägigen Kontexten verortet und viele formulierten pointierte Standpunkte zum Verhältnis von Geschlechter- und Behindertenpolitik.

Vor diesem Hintergrund ist die Interviewsequenz im Titel einerseits eine persönliche, an eigenen Erfahrungen mit gesellschaftlichen Barrieren orientierte Einschätzung. Andererseits kann sie als ein politisches Statement gele-

1 Die qualitative Längsschnittuntersuchung, auf die wir uns hier beziehen, wurde vom Hildegardis-Verein ebenfalls mit Mitteln der Contergan-Stiftung finanziert. Dafür danken wir im Namen der Universität Kassel ganz herzlich. Zum Programm vgl. Mentoring-Programm für Studentinnen mit Behinderung des Hildegardis-Vereins e.V.: http://www.hildegardisverein.de/mentoring- programm.html.

2 Im Folgenden wird immer wieder von „Frauen“ und „Männern“ (Mentorinnen und Mentoren) die Rede sein, weil diese Kategorisierungen für das untersuchte Programm eine wichtige Bedeutung haben. Wir folgen dieser Logik des untersuchten Zusammenhangs, statt die binäre Codierung der Sprache weiter aufzulösen, gleichwohl wir davon ausgehen, dass „Frauen“ und „Männer“ keine homogenen sozialen Gruppen bilden, „Weiblichkeit“ und „Männlichkeit“" soziale Konstruktionen sind und eindeutige, zweigeschlechtlich konnotierte Geschlechtsidentitäten eine (wissenschaftlich gestützte) Fiktion darstellen. 
sen werden: Welche gesellschaftlichen Diskriminierungsmechanismen haben wie viel Gewicht? Um das Gewicht und die Relationen unterschiedlicher Differenzkonstruktionen und Ungleichheitsdimensionen zu bestimmen, wählt die Studentin ein räumliches Bild. Zwischen Behinderung und Geschlecht sieht sie einen „meilenweiten“, also einen riesengroßen Abstand. Dies führt aus ihrer Sicht zu einer Nachordnung; Geschlecht, genauer gesagt „Frau“ kommt ,nach“ Behinderung.

Solche Überlegungen zur Gewichtung und möglichen Nachordnung von Ungleichheitsdimensionen werden auch in vielen anderen Interviews und in den Gruppendiskussionen der Studie formuliert (Bereswill, Pax \& Zühlke 2013: 140ff.). So wird über die Frage diskutiert, an wen Förderprogramme und politische Maßnahmen adressiert sein sollten: Ist es angemessen, nur Studentinnen zu fördern und damit ,Behinderung ' und ,Frau' als Dimensionen der sozialen Benachteiligung zu verknüpfen? Den meisten am Programm Teilnehmenden ist das Motiv der „,doppelten Benachteiligung“ geläufig, das behinderten Frauen zugeschrieben wird, und es wird immer wieder zum Aufhänger für Meinungsbildungsprozesse und eigene Verortungen (zur Kritik an einer additiven Sicht im wissenschaftlichen Diskurs vgl. Waldschmidt 2014a: 183f.; Walgenbach 2012: 45; Köbsell 2010: 27ff.). Dabei changieren die Auslegungen zwischen additiven Sichtweisen auf Mehrfachbenachteiligung, ausdrücklichen Betonungen einer Ungleichheitsdimension und der generellen Zurückweisung von Benachteiligung bis hin zur Hervorhebung der eigenen Privilegierung.

Den Schwerpunkt der Begleitstudie bildeten die Beziehungsgestaltung und die wechselseitigen Lernprozesse in den Tandems. Im vorliegenden Beitrag fokussieren wir hingegen die bereits angeklungene Frage nach den Deutungsmustern von Differenz und Ungleichheit, die im Rahmen des Programms und durch unsere Interventionen als Forscherinnen zur Sprache gebracht wurden. Diese sozialen Deutungsmuster begreifen wir in Anlehnung an Bogner, Littig und Menz (2009) als eine Form von prozessualem Expert*innenwissen, das von den Mentees, Mentor*innen in den Diskurs mitgebracht und zugleich während dieses Prozesses gemeinsam hergestellt wird. Von einem breit angelegten Verständnis des Expertenbegriffs ausgehend fokussieren wir damit einen sehr spezifischen Ausschnitt der ,gesellschaftliche Konstruktion von Wirklichkeit" (Berger \& Luckmann 1993), an deren intersubjektiver Aushandlung alle Teilnehmenden des Programms als Multiplikator*innen für Fragen der Inklusion mitwirken.

„Experten besitzen die Möglichkeit zur (zumindest partiellen) Durchsetzung ihrer Orientierungen. Experten zeichnen sich dadurch aus, dass sie maßgeblich bestimmen, aus welcher Perspektive und mithilfe welcher Begrifflichkeiten in der Gesellschaft über bestimmte Probleme nachgedacht wird. Genau diese Praxisrelevanz macht die Experten für viele empirische Forschungsprojekte und Forschungsfragen interessant" (Bogner, Littig \& Menz 2014: 14). 
In dem von uns untersuchten Programm treffen Personen in verschiedenen Funktionen aufeinander, die entsprechend unterschiedliche Formen von Wissen in den gemeinsamen Lernprozess einbringen: Erfahrungswissen, Fachwissen und Sonderwissen im Sinne einer weit über das gesellschaftliche Alltagswissen hinaus gehenden Auseinandersetzung mit gleichstellungspolitischen Fragen der Inklusion.

Im empirischen Teil dieses Beitrages beziehen wir uns auf Gruppendiskussionen, die wir im Rahmen der Studie geführt haben und bei denen Geschlecht von uns explizit als Ausgangsimpuls gewählt wurde. Forschungsleitend ist hierbei eine methodologische Perspektive, die Intersektionalität in Anlehnung an Gudrun-Axeli Knapp (2013: 251) und an eigene Überlegungen (Bereswill 2015: 226ff.) als sensibilisierendes Konzept heranzieht. Ausgehend davon, dass Geschlecht und Behinderung relationale soziale Kategorien der Differenz und Ungleichheit sind, deren wechselseitiges Verhältnis nicht abschließend bestimmt oder dingfest gemacht werden kann, werden die komplexen und widersprüchlichen Bedeutungszuschreibungen dieser sozialen Kategorien aus der Perspektive von Expert*innen rekonstruiert. ${ }^{3}$ Damit rückt eine mikrosoziologische Perspektive auf Differenz und Ungleichheit in den Vordergrund, die die vielfach geforderte Verknüpfung von Geschlechterforschung, Intersektionalitätsforschung und Disability Studies voran bringen möchte (Jacob, Köbsell \& Wollrad 2010: 7f.; Walgenbach 2012: 30ff.; Waldschmidt 2014a: 180ff.). Auch wenn die Gemeinsamkeiten der genannten Felder auf der Hand zu liegen scheinen, stellt sich die Frage nach den konkreten Schnittstellen und möglichen Erweiterungen der einen durch die andere Perspektive. Ziel des Beitrags ist die Konturierung einer empirisch begründeten Theoriebildung, in deren Mittelpunkt ein offenes Wechselverhältnis von Theorie und Empirie steht (Bereswill 2015: 227). Für eine an Intersektionalität orientierte rekonstruktive Forschung bedeutet dies die fortlaufende Reflexion auf die problematische und aus unserer Sicht zugleich unvermeidliche Setzung von als getrennt gedachten relationalen Kategorien wie Geschlecht und Behinderung. Deren strukturierende Wirkung für soziale Ordnung und für Ungleichheitskonstellationen $\mathrm{zu}$ untersuchen, ohne von Masterkategorien auszugehen, und sie zugleich als soziale und kulturelle Konstruktionen aufzudecken wird im vorliegenden Beitrag als Strategie der empirisch begründeten Theoriebildung angestrebt.

Wenn Geschlecht und Behinderung als relationale Kategorien aufgefasst werden, bedeutet dies, dass jede dieser beiden Kategorien ihre spezifische Ausprägung nicht nur im Wechselspiel mit der anderen (Behinderung-Geschlecht/Geschlecht-Behinderung), sondern auch im Wechselverhältnis mit weiteren wie Klasse, Milieu, soziale Herkunft, Alter, Sexualität erfährt. Die mit dieser Multidimensionalität einhergehende Steigerung der Komplexität von Untersuchungsansätzen (vgl. Bereswill 2014: 210) wird hier nicht weiter verfolgt, das damit verbundene Potenzial für eine differenzierte Auseinandersetzung mit Inklusion liegt aber auf der Hand. 
Im folgenden Abschnitt nehmen wir Bezug auf die bereits eingeführte Studie, auf deren Daten wir uns in diesem Text beziehen. Wir erläutern die Entscheidung, Ausschnitte aus Gruppendiskussionen $\mathrm{zu}$ analysieren (2.). Anschließend rekonstruieren wir Deutungsmuster zum Verhältnis von Geschlecht und Behinderung, wobei die Bedeutung des Körpers für diese Relation hervortritt (3.). Im Ausblick nehmen wir die einleitenden Überlegungen zur Verknüpfung von Geschlechterforschung, Intersektionalitätsforschung und Disability Studies wieder auf und konkretisieren diese im Hinblick auf unsere Ergebnisse (4.).

\section{Der qualitative Untersuchungsansatz}

In der Begleitstudie haben wir zu Beginn und nach Ende des Programms ein themenzentriertes, diskursiv angelegtes Leitfadeninterview mit den Teilnehmenden erhoben (Kruse 2014; Bogner/Littig/Menz 2005; Witzel 2000). Jede der drei Kohorten des Programms wurde zudem zur Teilnahme an Gruppeninterviews eingeladen, die im ersten Jahr auf Fragen von Mentoring als Konzept und in den folgenden Jahren auf Gleichstellungsfragen fokussiert waren. Alle Daten wurden mit rekonstruktiven Methoden ausgewertet, indem zunächst Kategorien aus dem Material und zu einem späteren Zeitpunkt Feinanalysen ausgewählter Textsequenzen in Interpretationsgruppen durchgeführt wurden (Strauss 1994; Wernet 2000; Meuser \& Nagel 2005). Neben Fallanalysen der interviewten Tandems (Bereswill, Pax \& Zühlke 2013: 30139) wurde eine themenzentrierte Auswertung der Interviews durchgeführt, so dass der Bedeutung von Geschlecht und Behinderung sowohl fallspezifisch als auch fallübergreifend nachgegangen werden konnte.

Auf Grundlage der ersten Interviewauswertungen wurden Impulse für Gruppeninterviews entwickelt, in denen die Teilnehmer*innen einen selbstläufigen Diskurs über ihre Erfahrungen und Einschätzungen entfalten konnten. Diese methodische Erweiterung ermöglichte es uns, bestimmte Deutungsmuster (z.B. zu ,gutem ' Mentoring oder zu gleichstellungspolitischen Fragen) in einem mehrfach kontrastiven Vergleich heraus zu arbeiten - über verschiedene Einzelinterviews und Gruppendiskussionen hinweg. Dabei wird in den Interviews wie auch in den Gruppendiskussionen nachvollziehbar, wie die eigenen Erfahrungen in einem ständigen Vergleich mit denen anderer reflektiert, bestätigt und überdacht werden.

Zwei dieser Gruppeninterviews in zwei verschiedenen Jahrgängen bilden die Datenbasis für den vorliegenden Beitrag. Insgesamt nahmen 28 Personen mit und ohne Behinderung daran teil. Die genaue Zusammensetzung der Gruppen wird aus Gründen des Datenschutzes nicht weiter ausgeführt. Da in den Einzelinterviews vermehrt das Bild der additiven Benachteiligung von 
Frauen mit Behinderung herangezogen wurde und das Programm zudem ein Frauenförderprogramm war, entschlossen wir uns dazu, den Fokus auf Frauen als eine benachteiligte Gruppe weiter zuzuspitzen und setzten die folgende einseitig formulierte These an den Anfang der Diskussionen:

„Studentinnen und Akademikerinnen mit Behinderungen stehen beim Erreichen von beruflichen Zielen enormen Hürden gegenüber. Diese Herausforderungen betreffen Frauen stärker als Männer. Wie sehen Sie das?“

Im Anschluss an diese eindimensionale Sicht auf Geschlecht entspannen sich lebhafte und verwickelte Auseinandersetzungen, aus denen wir im Folgenden kurze Ausschnitte aufgreifen. Wir fokussieren auf thematische Aspekte und diskursive Konstellationen, in denen das Verhältnis von Geschlecht und Behinderung explizit oder implizit zur Diskussion gestellt wird und binden die einzelnen Statements nur vage an die Rolle der Sprecher*innen im Programm zurück. Auf die weitere Diversifizierung der Generierung von Wissen im Interaktionsprozess der Gruppe wird verzichtet, um den Aufmerksamkeitsfokus für die zentralen gemeinsamen Deutungsmuster der verschiedenen Sprecher*innen zu schärfen.

\section{Komplexe Relationen}

Die Forschungsfrage nach den spezifischen Verflechtungen, Verknüpfungen und Verdeckungen von Geschlecht und Behinderung steht im Kontext von leistungsgeprägten akademischen Karrieremustern. Wir rekonstruieren im Folgenden Selbstthematisierungen und Deutungsmuster von akademisch sozialisierten Akteur*innen, deren Auseinandersetzung mit Leistungserwartungen und Leistungsfähigkeit im Bildungssystem von großer Bedeutung für ihren Austausch miteinander und für ihre Selbstpositionierungen sowohl in den Einzel- als auch in den Gruppeninterviews ist. Die Bedeutung von Geschlecht und Behinderung entfaltet sich demnach einerseits im Wechselspiel von biographischen Prozessen und situativen Interaktionen, andererseits wird sie im Kontext des meritokratischen Prinzips des akademischen Systems hervor gebracht. Diese Dynamik wird im folgenden Abschnitt anhand von Passagen aus den Gruppendiskussionen nachvollziehbar. 


\section{1. „Das war eher ein positiver Diskriminierungsgrund“- Behinderung als Chance zum Aufstieg}

Im Zusammenhang mit dem Thema Diskriminierung spricht eine Mentorin über ihre Herkunftsfamilie und betont dabei den Kontrast zwischen dem Werdegang ihrer Geschwister und ihrem eigenen:

„Ich komme aus einer (Gewerbe-)Familie und meine Geschwister haben dort auch gearbeitet und weil ich eine Behinderung hatte, konnte ich da nicht so richtig den Platz finden und ich glaube, das war eher ein positiver Diskriminierungsgrund, dass ich die höhere Schule besuchen durfte, um der Situation mit Behinderung zu leben anders gewappnet zu sein.“

Die Schilderung vermittelt ein widersprüchliches Bild. Indem die Teilhabe an der familiären Wirtschaftsgemeinschaft verwehrt bleibt, öffnet sich zugleich der Weg zu höherer Bildung, mit dem der Ausschluss aus dem Familienzusammenhang als ,positive Diskriminierung“ kompensiert und dabei zugleich die Grenzen der eigenen Herkunft überschritten werden können. Eine Mentee, die an der gleichen Gruppendiskussion mitwirkt, spricht kurze Zeit später ebenfalls über die Bedeutung von Familienbeziehungen für ihren Werdegang. Auch hier wird der Kontrast zwischen dem eigenen Bildungsweg und dem der Geschwister hervorgehoben und damit unterstrichen, dass es sich im familiären Kontext um einen Bildungsaufstieg handelt.

„Sie wussten, dass ich für so eine Behindertenwerkstattkarriere viel zu intelligent bin und da hab ich als einzige meiner Geschwister es ergriffen zu studieren und das war schon wichtig, weil anderweitig wäre mir wahrscheinlich nur wirklich diese Behindertenlaufbahn geblieben."

Mit Bezug zur Frage nach Deutungsmustern von Geschlecht und Behinderung tritt in dieser wie auch in der Passage zuvor Behinderung in den Vordergrund des manifesten Diskurses. Dabei wird greifbar, was Swantje Köbsell zum Verhältnis von Behinderung und Geschlecht betont: während Geschlecht (immer noch) mit einer homogenen sozialen Gruppe assoziiert wird, „wird mit $>$ behindert $<$ allerdings keine homogene, klar abgrenzbare Gruppe von Menschen bezeichnet" (Köbsell 2010: 21, Hervorheb. i. Orig.). Damit verbunden sind Abgrenzungen zwischen unterschiedlichen Formen und Folgen von Behinderung, auf die die Sprecherin der zuletzt zitierten Passage anspielt. Bemerkenswert ist dabei der starke Kontrast, den die Sprecherin zwischen dem eigenen gesellschaftlichen Ort als Akademikerin und den Orten, die für Menschen mit einer „Behindertenlaufbahn“ vorgesehen sind, zur Sprache bringt. Der Akademikerinnenkarriere wird eine Karriere als stigmatisiert gegenübergestellt. Dabei korrespondiert die Abgrenzung unterschiedlicher Bildungsräume mit der Distinktion zugeschriebener Fähigkeiten: die eigene ausgeprägte Intelligenz und mit Behinderung assoziierte kognitive Beeinträchtigungen schließen sich aus. Diese Abgrenzung unterstreicht zu- 
gleich, dass Behinderung und wissenschaftliche Leistung sich nicht ausschließen, gesellschaftlich aber nicht vorgesehen sind. Die damit verbundene Erfahrung als eine Ausnahme darzustellen, wird in der folgenden Feststellung durch eine Mentee zur Sprache gebracht:

„Da wir alle, die wir hier sitzen, einen besonderen Background haben und eigene Wege gegangen sind, akademische Wege, die aber auch vielleicht nicht der Normalität der normalen Frau mit Behinderung entsprechen."

Das „wir alle“ betont die Gemeinsamkeit der Gruppe, vermutlich auch über die konkrete Gruppendiskussion hinaus auf das gesamte Programm bezogen. Geteilt wird der „besondere“ Hintergrund, der in „eigenen“, ,akademischen“ Wegen gesehen wird. Damit wird eine Abweichung verbunden: von der „Normalität der normalen Frau mit Behinderung“. Die sprachliche Verdoppelung und Bedeutungsverschiebung von „Normalität“ als einer Ordnungsvorstellung zu „normal“" als Eigenschaft einer Person konstruiert eine doppelte Abweichung: ,behindert' weicht von ,nicht-behindert" ab und ,nicht-normal behindert" von ,normal behindert'. Welche Bedeutung „Frau“ dabei erfährt, bleibt offen. Möglicherweise wird der eigene Weg als Akademikerin mit Behinderung auch als eine Abweichung von der gängigen Geschlechterordnung in diesem Feld eingeschätzt. Es handelt sich um eine verwickelte Argumentation, in der die "Thematisierung von Differenz" eng mit der Entfaltung von „Normalisierungsmacht“ verknüpft ist (vgl. Wacker 2014: 234), wobei der eigene Werdegang als Abweichung gekennzeichnet wird. Welche „Normalität“ Akademikerinnen hinter sich lassen, bleibt an dieser Stelle unausgesprochen, ist aber in den vorherigen Beiträgen in Bildern wie der „Behindertenlaufbahn“ und der „Werkstatt" angeklungen. Gegen dieses Bild wird die eigene Leistungsfähigkeit gesetzt, die zugleich immer wieder als Kompensation thematisiert wird. Hierbei gewinnt der Körper seine Bedeutung als Bezugspunkt für , andere‘ Leistungsfähigkeiten.

\section{2. „Ob das frauenspezifisch ist “-Geschlecht und Behinderung als ausgleichende Größen}

Sehr viel später im Verlauf des vorher zitierten Gruppeninterviews kommt eine andere Mentee erneut auf die Bedeutung von Geschlecht zu sprechen. Sie zögert zunächst, wenn sie sagt ,ich weiß nicht, ob das frauenspezifisch ist, also ob das mit Geschlecht zu tun hat", bevor sie fortfährt:

„Aber ich kenne dieses Gefühl körperliche Defizite auszugleichen mit guten Leistungen, wo man überzeugen kann und in Studienleistungen, Noten und beruflichen Leistungen, wo man keine körperliche Arbeit braucht kann man dann gut Erfolge erzielen mit denen man versuchen kann zu kompensieren, auch für sich selber, um zu zeigen ich kann was womit 
ich überzeugen kann egal ob ich behindert bin oder nicht, ob das frauenspezifisch ist oder ob das für alle behinderten Akademiker gilt.“

Hier wird das Selbstbewusstsein über die eigenen Fähigkeiten vor dem Hintergrund eines Gefühls des Mangels thematisiert. Dieser Mangel wird am eigenen Körper und an den Anforderungen körperlicher Arbeit festgemacht. Beschrieben wird eine Kompensationsdynamik: wo keine körperliche Arbeit erwartet wird, tritt die Behinderung in den Hintergrund - ,egal ob ich behindert bin oder nicht" - und die eigene Leistungs- und Überzeugungskraft rückt in den Vordergrund. Dieser Entwurf einer vom Körper losgelösten Dimension der Anerkennung durch Andere unterstreicht zugleich die herausragende Bedeutung des Körpers für die soziale Ordnung, auf die nicht nur im Zusammenhang der Disability und Gender Studies immer wieder hingewiesen wird.

„Die Sichtbarkeit bzw. allgemeiner, die unmittelbare Wahrnehmbarkeit von Körpern und ihrer Aktionen ist von besonderer Bedeutung für das Herstellen jeder Interaktionsordnung und für den Verlauf von Kategorisierungsprozessen in der Interaktion von Behinderung“" (Groenemeyer 2014: 156; vgl. auch Goffman 1999).

In solchen Kategorisierungsprozessen wirken Konstruktionen von Behinderung und solche von Geschlecht ineinander (Köbsell 2010: 22ff.) und bringen dabei quasi-natürliche weibliche, beeinträchtigte oder intakte Körper zur Geltung.

Das Bild einer vom Körper unabhängigen Leistungsfähigkeit, das die Mentee in der zuvor zitierten Passage entwickelt, steht im deutlichen Gegensatz zum Bild eines weiblich konnotierten Körpers, der als unberechenbar und für Karrieren hinderlich betrachtet wird. Diese Dimension des Körpers wird im Zusammenhang mit der Vereinbarkeitsfrage thematisiert. So greift eine Mentee auf das Bild der Doppelbelastung zurück, wenn sie sagt ,ich glaube, dass Frauen irgendwie eine Doppelbelastung haben" und dies anschließend weiter ausführt, indem sie einen grundlegenden Unterschied konstruiert:

"Weil eine Frau bewirbt sich mit einem Job und dann kommt die Frage haben Sie denn schon Kinder und wenn es eine junge Frau ist, besteht die Gefahr, dass sie ausfällt aus Schwangerschaftsgründen oder so und bei Behinderten geht es halt nur um die Krankheitsgeschichte."

Es handelt sich um den bemerkenswerten Vergleich zwischen einer typisierten ,junge(n) Frau“, die möglicherweise schwanger wird und geschlechtlich nicht weiter verorteten „Behinderten“, in deren Bewerbung „nur“ die „Krankheitsgeschichte“ thematisiert wird. Sind „Behinderte“ gegenüber Frauen ohne Behinderung deshalb im Vorteil oder im Nachteil? Oder sind „Behinderte“ und (potentiell) Schwangere eher gleich gestellt, weil in beiden Fällen auf die Einschränkung von Einsatzmöglichkeiten geschaut wird? Die 
Sprecherin lässt diese Frage offen, wobei auffällt, dass sie rein sprachlich „Frau“ und „Behinderte“ und nicht „Frau“ und ,behinderte Frau“ miteinander vergleicht. Diese sprachliche Neutralisierung passt dazu, dass sie feststellt, Menschen mit Behinderung würden in der Wahrnehmung von Entscheidungstragenden auf diese reduziert. Der Vergleich verweist aber trotz der sprachlichen Neutralisierung auf Weiblichkeitszumutungen, indem ,eine Familiengründung für als körperlich behindert kategorisierte Frauen gesellschaftlich tendenziell nicht als eine Option gesehen wird" (Walgenbach 2012: 31). Diese Diskreditierung wird im Redebeitrag der Mentee nur zwischen den Zeilen thematisiert und es bleibt unklar, wer gegenüber wem aufgrund welcher Erwartungen und Zuschreibungen im Vor- oder Nachteil ist.

Auf jeden Fall lenkt auch dieses Deutungsmuster den Blick auf die Bedeutung des funktionierenden oder funktionalen Körpers für Konstruktionen von Geschlecht und Behinderung. Familiengründung wird dabei mal als hinderlich und mal als ein Vorteil bewertet, wenn zum Beispiel eine Mentorin feststellt, ,wir Frauen haben im Zweifelsfall die Möglichkeit in die Familie zu flüchten“".

Die Möglichkeit des Rückzugs betrachtet die Sprecherin zugleich als ein Privileg von Frauen im Vergleich mit Männern, die den Rückzug in die Familie nicht in Betracht ziehen könnten: „Das ist natürlich etwas, das können Männer für sich nicht gestalten“. So entsteht über die verschiedenen Redebeiträge hinweg das Bild einer widersprüchlichen Geschlechterordnung, in der Weiblichkeit und Männlichkeit - als körpergebundene Phänomene - mal mit Privilegien und mal mit Nachteilen verknüpft scheinen. Behinderung - als ebenfalls körpergebunden - wirkt an diesen wechselnden Prozessen von Über- und Unterordnungen mit und wird ebenfalls mal als Malus und mal als Bonus erlebt. Dabei strukturieren implizite wie explizite Vergleiche die Positionen und Argumente, wobei mal ,Frauen', mal ,Frauen' und ,Behinderte“ und mal ,Frauen' und ,Männer' verglichen werden. Im folgenden Abschnitt wird die Auseinandersetzung mit Männlichkeit fokussiert, in deren Kontext sich bestimmte Argumentationsfiguren zum Zusammenhang von Behinderung und Geschlecht weiter zuspitzen.

\section{3. „Dass das männliche Selbstbild dann ins Wanken kommt" - Weiblichkeit als Bonus}

Dass wirkmächtige Konstruktionen von Geschlechterdifferenz den gesellschaftlichen Umgang mit Behinderung (und damit auch mit Inklusion) mitstrukturieren, ist vielen Teilnehmer*innen unserer Untersuchung präsent. So wird beispielsweise wiederholt thematisiert, dass Weiblichkeit und Hilfebedürftigkeit gesellschaftlich als kongruent und vor allem unauffällig, Männ- 
lichkeit und Hilfebedürftigkeit hingegen als inkongruent und abweichend bewertet werden:

„Männer, die Hilfe brauchen, da steht die Hilfsbedürftigkeit im Vordergrund, denn wenn einer nichtbehinderten Frau was runterfällt kann es auch sein, es hebt jemand für sie auf, das es eben zu dem Frauenbild auch ein Stück weit gehört sich mal helfen oder unterstützen zu lassen oder das niemand sich was dabei denkt, wenn Frauen zum Beispiel auch untergehakt laufen, aber zwei Männer, die also ein Mann der einen anderen Mann führt, das hat schon irgendwie wieder so ein bisschen was in die homosexuelle Ecke.“

Hier wird ausdrücklich betont, dass Frauen gegenüber Männern im Vorteil seien. Brauchen Männer Hilfe, werden sie auf ihre Behinderung zurückgeworfen und müssen zudem mit Zuschreibungen von (verweiblichter) Homosexualität rechnen, wenn andere Männer sie körperlich berühren und führen. Der männlich konnotierte Körper wird dabei als verletzungsoffen und sexualisiert dargestellt - ein Bild, das eher im Zusammenhang mit weiblich konnotierten Körpern geläufig ist. Zugespitzt bedeutet das, stereotype und heteronormative Repräsentationen von Weiblichkeit, besser gesagt von Geschlechterdifferenz, schützen vor Herabwürdigung aufgrund von Behinderung.

Dieser Redebeitrag veranschaulicht wie das „Sameness Taboo“ (Rubin 1997) im Zusammenspiel von Behinderung und Geschlecht wirkt. Weiblichkeit und Vulnerabilität sind passförmige, Männlichkeit und Vulnerabilität sind inkongruente Verknüpfungen und führen $\mathrm{zu}$ entsprechend differenten Konsequenzen für Frauen und Männer (auch dann, wenn sie nicht mit Behinderungen leben). Dieses Muster betont auch Swantje Köbsell, stellt allerdings fest, es gäbe für Männer die Möglichkeit, sich gesellschaftlichen Geschlechternormen über die Berufsrolle stärker anzunähern als dies für Frauen der Fall sei (Köbsell 2010: 22).

Im Gegensatz zu dieser Einschätzung betonen die Teilnehmerinnen der Gruppendiskussion, dass Männlichkeitszumutungen wie die des erfolgreichen Erwerbsarbeiters die Verletzungsoffenheit von Männern mit Behinderung zuspitzen und machen dies am vulnerablen Körper fest. Deutlich wird hierbei nicht nur, dass „Geschlechterordnungen in körperlichen Praktiken her- und dargestellt, angeeignet, stabilisiert oder verworfen werden" (Gugutzer 2014: 93), sondern auch, dass Geschlecht in Relation zu Behinderung eine Bedeutungsverschiebung erfährt. Diese Sichtweise auf eine vorherrschende Geschlechterordnung spitzt sich weiter zu, wenn, wie in der folgenden Passage, „der Mann als Leistungsträger“ in den Blick genommen wird:

„Das find ich interessant gerade auch wieder es ja immer noch das Bild gibt der Mann als Leistungsträger, als Ernährer der Familie, was das möglicherweise für vielleicht noch viel krassere Identitätsbedrohung für behinderte Männer bedeutet.“

Hier stellt eine Mentee fest, das gängige Bild von Männlichkeit sei auch gegenwärtig noch der „Ernährer“. Dabei verkehrt die gesellschaftliche Ge- 
schlechterhierarchie sich auch aus ihrer Sicht ins Gegenteil: Weiblichkeitsklischees, die Frauen auf abhängige Wesen reduzieren, wirken weniger zerstörerisch auf das Selbstempfinden als Männlichkeitsklischees, die Männern Unabhängigkeit und Unverletzlichkeit abverlangen. Erfahren Männer mit Behinderung demnach gravierendere Benachteiligungen als Frauen mit Behinderung? Diese Frage wird auch von anderen Teilnehmerinnen aufgenommen. In der folgenden Passage plädiert eine Mentee allerdings dafür, die Benachteiligungen von Männern und von Frauen aus dieser Diskussion nicht gegeneinander auszuspielen.

„Wenn sie [Männer] noch zusätzlich eine Behinderung haben, kann ich mir vorstellen, dass es mit dem Männerbild, also dass das männliche Selbstbild dann ins Wanken kommt, weil der Mann traditionell der starke Verdiener sein muss und Ernährer und deswegen ein besonderer Nachteil für Männer auch gegeben ist, wenn Männer mit Behinderung auf Jobsuche gehen, aber das mindert natürlich nicht den Nachteil von Frauen, also ich denke, ich wollte nur ja aufwiegen, dass es dadurch wahrscheinlich ähnliche Nachteile gibt.“

Hier wird fraglos vorausgesetzt, dass Behinderung Männlichkeit schwächt und implizit an das Bild einer intakten Männlichkeit angeknüpft. Auch wenn dieses Argument sofort relativiert wird, wird zwischen den Zeilen trotzdem deutlich, welchen Bezugspunkt der Vergleich hat: eine Version dominanter, „starker“ Männlichkeit, an der sowohl andere Männlichkeiten als auch Weiblichkeiten gemessen werden. Bemerkenswert ist dabei, dass die Vergleiche zwischen Frauen und Männern darauf hinauslaufen, dass die Verletzungsmacht, die mit Männlichkeit verbunden ist, Männer härter trifft als Frauen. Die damit verbundene Version hegemonialer Männlichkeit (Connell 1995) erinnert an eine Überlegung, die Erving Goffman in seinem Text Stigma bereits in den 1960er Jahren formuliert hat.

„Zum Beispiel gibt es in einem gewichtigen Sinn nur ein vollständig akzeptables männliches Wesen in Amerika: ein junger, verheirateter, weißer, städtischer, nordstaatlicher, heterosexueller Vater mit Collegebildung, voll beschäftigt, von gutem Aussehen, normal in Gewicht und Größe und mit Erfolg im Sport“" (1999: 158, Hervorheb. i.Orig.).

Dass diese „gängige Grundannahme“ einer weißen Mittelschichtmännlichkeit längst nicht überwunden ist (vgl. Waldschmidt 2014a: 182), wird von den Teilnehmer*innen unserer Untersuchung explizit formuliert. Die Schlussfolgerung, dass das Zusammenspiel von Behinderung und Geschlecht Frauen gegenüber Männern tendenziell zum Vorteil oder zumindest zu weniger Nachteil gerät, ist aus Sicht der Diskutantinnen nachvollziehbar. Ob der „Faktor Frau“ sich aber tatsächlich als Bonus erweist, ist eine offene Frage, der wissenschaftlich erst einmal weiter nachzugehen wäre. 


\section{Der Körper als Vermittlungsmodus von Differenz und Ungleichheit}

Zusammenfassend ist festzustellen, dass die wechselseitigen Bedeutungszuschreibungen von Geschlecht und Behinderung, die in den untersuchten Deutungsmustern erkennbar werden, keine abschließenden Aussagen über die Gewichtung gesellschaftlicher Diskriminierungsmechanismen erlauben, gleichwohl verschiedene Positionen in den Gruppendiskussionen dies aus der Perspektive der subjektiven Erfahrungen nahe legen. Vielmehr ist Katharina Walgenbach darin zuzustimmen, dass Geschlecht und Behinderung nicht voneinander getrennt werden können und „dies für Frauen mit Behinderungen oft eine Verweigerung von Geschlechtsidentität, biologischer Reproduktion oder Heterosexualität" bedeute (2010: 33; vgl. auch: Raab 2012: 78). Diese Einschätzung wird durch unsere Ergebnisse erweitert, indem die Verflechtungen von Behinderung mit hegemonialer Männlichkeit deutlich und deren Einfluss auf Männer wie Frauen anschaulich wurde. Hier zeigen sich aus unserer Sicht bislang nicht genutzte Potenziale der Männlichkeitsforschung für die Weiterentwicklung und Verknüpfung von Forschungsansätzen zu den Relationen von Geschlecht und Behinderung in Geschlechterforschung, Intersektionalitätsdebatten und Disability Studies.

Unsere Rekonstruktionen haben zudem gezeigt, dass die Wirkmacht von Zuschreibungen, Erwartungen und Normalitätsmaßstäben sich am und durch den Körper manifestiert. Gabriele Winker und Nina Degele (2009) haben schon vor einigen Jahren vorgeschlagen, den Körper analog zu Klasse, ,Ras$\mathrm{se}^{\text {' }}$ und Geschlecht als eine vierte Strukturkategorie zu erfassen und sprechen von Bodyismen: „Unter Bodyismen verstehen wir entsprechend der Wichtigkeit der Strukturkategorie Körper Herrschaftsverhältnisse zwischen Menschengruppen aufgrund körperlicher Merkmale“ (2009: 51). Dieses herrschaftskritische Argument ist insbesondere für die Bedeutung des leistungsfähigen Körpers in der postfordistischen Gesellschaft plausibel. Trotzdem plädieren wir dafür, den Körper nicht als Strukturkategorie zu fassen. Unsere Überlegungen lauten vielmehr, dass

„die Bedeutung des Körpers darin liegt, dass er Ungleichheitskonstellationen immer schon durchzieht. Daraus ergibt sich die Frage, was die spezifische Leistung einer intersektionalen Untersuchungsperspektive auf den Körper ausmacht. Könnte die systematische Aufmerksamkeit für den Körper zur Öffnung und Dezentrierung von eindimensionalen Masterkategorien beitragen?" (Bereswill 2015: 222).

Aus diesem Blickwinkel ist der Körper keine Strukturkategorie, sondern ein Vermittlungsmodus verschiedener Ungleichheitsdimensionen, wie sie sich beispielsweise empirisch-konkret im Deutungsmuster einer vulnerablen, durch Weiblichkeitszuschreibungen bedrohten Männlichkeit manifestieren 
(vgl. Bereswill 2007). Hier zeigen sich systematische Verbindungen zwischen den Disability Studies mit ihrer gezielten Kritik ,der normativen Ordnung des Somatischen“ (Raab 2012: 71) und soziologischen, geschlechtersowie queertheoretischen Ansätzen, die den Körper als performativ hervorgebracht analysieren (vgl. Schroer 2005; Wagels 2008; Villa 2010; Manz 2013).

Zugleich verweist die Auseinandersetzung mit der Bedeutung des Körpers für Behinderung auf die unhintergehbare „ontologische Unsicherheit“ (Winker \& Degele 2009: 51), die durch Vorstellungen des perfekten, leistungsfähigen Körpers verdeckt gehalten wird. Swantje Köbsell plädiert deshalb dafür,

„den beeinträchtigen Körper in den Diskurs der Disability Studies einzubeziehen, um hierzu einen Gegenwentwurf zu liefern und deutlich zu machen, welche gesellschaftlichen Mechanismen am Prozess der Behinderung beteiligt sind“ (2010: 28).

Das gilt auch für Geschlechterkonstruktionen, deren Körpergebundenheit dazu herausfordert, den Körper als sozialen Akteur zu erfassen, ohne seine Materialität und Vulnerabilität zu leugnen.

Werden die Ergebnisse unserer Untersuchung und deren theoretische Reflexion auf die Frage nach dem „Geschlecht der Inklusion“ bezogen, die die Herausgeber*innen dieses Jahrbuchs aufgeworfen haben, ist festzustellen, dass gesellschaftliche Inklusion und Exklusion höchst vergeschlechtlichte Prozesse sind, dass dabei aber keine eindeutigen und ,typischen' Muster der Geschlechterdifferenz, sondern vielmehr komplexe Bedeutungszuschreibungen und Bedeutungsverschiebungen erkennbar sind. Es handelt sich vielmehr um widersprüchliche und widersinnige Dynamiken, die weiter ausgelotet werden müssen.

\section{Literatur}

Bereswill, Mechthild (2007): Sich auf eine Seite schlagen. Die Abwehr von Verletzungsoffenheit als gewaltsame Stabilisierung von Männlichkeit. In: Bereswill, Mechthild/Meuser, Michael/Scholz, Sylka (Hrsg.): Dimensionen der Kategorie Geschlecht: Der Fall Männlichkeit? Münster: Westfälisches Dampfboot, S. 191-118.

Bereswill, Mechthild (2015): Komplexität steigern. Intersektionalität im Kontext von Geschlechterforschung. In: Bereswill, Mechthild/Degenring, Folkert/ Stange, Sabine (Hrsg.) (2015): Intersektionalität und Forschungspraxis. Wechselseitige Herausforderungen, Band 43 der Schriftenreihe der Sektion Frauen- und Geschlechterforschung in der 
Deutschen Gesellschaft für Soziologie. Münster: Westfälisches Dampfboot, S. 210-230.

Bereswill, Mechthild/Pax, Rafaela M./Zühlke, Johanna (2013): Mentoring als Möglichkeitsraum. Erfahrungen der Teilnehmerinnen und Teilnehmer eines Mentoring-Programms für Studentinnen mit Behinderungen. Kassel: University Press.

Berger, Peter L./Luckmann, Thomas (1993/1969): Die gesellschaftliche Konstruktion von Wirklichkeit. Eine Theorie der Wissenssoziologie. Frankfurt am Main: Fischer.

Bogner, Alexander/Littig, Beate/Menz, Wolfgang (Hrsg.) (2005): Das Experteninterview. Theorie, Methode, Anwendung. Wiesbaden: VS Verlag für Sozialwissenschaften.

Bogner, Alexander/Littig, Beate/Menz, Wolfgang (2014): Interviews mit Experten. Eine praxisorientierte Einführung. Wiesbaden: Springer VS Verlag für Sozialwissenschaften.

Connell, Robert W. (1995): Masculinities. Cambridge: Polity Press.

Goffman, Erving (1999/1975): Stigma. Über Techniken der Bewältigung beschädigter Identität. Frankfurt/Main: Suhrkamp.

Groenemyer, Axel (2014): Soziale Praxis- Diskurse- Erfahrung. Behinderung im Problematisierungsprozess. In: Soziale Probleme. Zeitschrift für soziale Probleme und soziale Kontrolle 25, 2, S.150-171.

Gugutzer, Robert (2014): Leibliche Praktiken der Geschlechterdifferenz. Eine Neophänomenologische Kritik der Praxeologie des Körpers in handlungstheoretischer Absicht. In: Behnke, Cornelia/Lengersdorf, Diana/Scholz, Sylka (Hrsg.): Wissen-Methode-Geschlecht: Erfassen des fraglos Gegebenen. Wiesbaden: Springer VS Verlag für Sozialwissenschaften, S. 91-106.

Hildegardis-Verein e.V. (o.A.): Mentoring-Programm für Studentinnen mit Behinderung. http://www.hildegardis-verein.de/mentoring-programm. html [Zugriff: 30.3.2015].

Knapp, Gudrun-Axeli (2013): Über Kreuzungen: zu Produktivität und Grenzen von „Intersektionalität“ als „Sensitizing Concept“. In: Bereswill, Mechthild/Liebsch, Katharina (Hrsg.): Geschlecht (re)konstruieren. Zur methodologischen und methodischen Produktivität der Frauen- und Geschlechterforschung, Band 38 der Schriftenreihe der Sektion Frauen- und Geschlechterforschung in der Deutschen Gesellschaft für Soziologie. Münster: Westfälisches Dampfboot, S. 210-230.

Kruse, Jan (2014): Qualitative Interviewforschung. Ein integrativer Ansatz. Weinheim: Beltz Juventa.

Köbsell, Swantje (2010): Gendering Disability. Behinderung, Geschlecht und Körper. In: Jacob, Jutta/Köbsell, Swantje/Wollrad, Eske (Hrsg.): Gendering Disability. Intersektionale Aspekte von Behinderung und Geschlecht. Bielefeld: transcript, S. 17-34. 
Manz, Ulrike (2013): Praktiken und Geschlecht. Methodologische Überlegungen zur Produktivität des practice turn. In: Bereswill, Mechthild/Liebsch, Katharina (Hrsg.): Geschlecht (re)konstruieren. Zur methodologischen und methodischen Produktivität der Frauen- und Geschlechterforschung, Band 38 der Schriftenreihe der Sektion Frauen- und Geschlechterforschung in der Deutschen Gesellschaft für Soziologie. Münster: Westfälisches Dampfboot, S. 115-133.

Meuser, Michael/Nagel, Ulrike (2005): ExpertInneninterviews. Vielfach erprobt, wenig bedacht. In: Bogner, Alexander/Littig, Beate/Menz, Wolfgang (Hrsg.): Das Experteninterview. Theorie, Methode, Anwendung. Wiesbaden: VS Verlag für Sozialwissenschaften, S. 71-93.

Raab, Heike (2012): Doing Feminism: Zum Bedeutungshorizont von Geschlecht und Heteronormativität in den Disability Studies. In: Rathgeb, Kerstin (Hrsg.): Disability Studies. Kritische Perspektiven für die Arbeit am Sozialen. Wiesbaden: Springer VS Verlag für Sozialwissenschaften, S.69-89.

Schroer. Markus (Hrsg.) (2005): Soziologie des Körpers. Frankfurt/Main: Suhrkamp.

Strauss, Anselm L. (1994): Grundlagen qualitativer Sozialforschung. München: Wilhelm Fink.

Villa, Paula Irene (2010): Subjekte und ihre Körper. Kultursoziologische Überlegungen. In: Wohlrab-Sahr, Monika (Hrsg.): Kultursoziologie. Paradigmen - Methoden - Fragestellungen. Wiesbaden: VS Verlag für Sozialwissenschaften, S. 252-271.

Wacker, Elisabeth (2014): Verwobene Behinderungsprobleme. Diversität und Inklusivität als Spagat und Zwickmühle. In: Soziale Probleme. Zeitschrift für soziale Probleme und soziale Kontrolle 25, 2, S.232-267.

Wagels, Karen (2008): „Der hatte 'ne Position - und ich hatte keine“. Regulierungsweisen von Geschlecht in Erwerbsarbeitskontexten. In: Marburger Gender-Kolleg (Hrsg.): Geschlecht Macht Arbeit. Münster: Westfälisches Dampfboot, S. 136-151.

Waldschmidt, Anne (2014a): Macht der Differenz. Perspektiven der Disability Studies auf Diversität, Intersektionalität und soziale Ungleichheit. In: Soziale Probleme. Zeitschrift für soziale Probleme und soziale Kontrolle 25, 2, S. 173-192.

Waldschmidt, Anne (2014b): Jenseits der doppelten Diskriminierung? In: Löw, Martina (Hrsg.): Vielfalt und Zusammenhalt. Verhandlungen des 36. Kongresses der Deutschen Gesellschaft für Soziologie in Bochum und Dortmund 2012. Frankfurt/Main: Campus, S. 871-883.

Walgenbach, Katharina (2012): Gender als interdependente Kategorie. In: Walgenbach, Katharina/ Dietze, Gabriele/Hornscheidt, Lann/Palm, Kerstin (Hrsg.): Gender als interdependente Kategorie. Neue Perspektiven auf 
Intersektionalität, Diversität und Heterogenität. Opladen/Berlin/Toronto: Barbara Budrich, S. 23-64.

Wernet, Andreas (2000): Einführung in die Interpretationstechnik der objektiven Hermeneutik. Qualitative Sozialforschung, Bd. 11. Opladen: Leske + Budrich.

Winker, Gabriele/Degele, Nina (2009): Intersektionalität. Zur Analyse sozialer Ungleichheiten. Bielefeld: transcript.

Witzel, Andreas (2000): Das problemzentrierte Interview. In: Forum Qualitative Sozialforschung /www.qualitative-research.net/index.php/fqs/article/ viewArticle /1132/2519 [Zugriff: 18.3.2015]. 